gresión logística no reveló diferencias en las tasas de mortalidad infantil durante los dos períodos examinados. Se encontró un descenso sorprendente del riesgo de muerte infantil en hogares con mala infraestructura, compensado por un riesgo mayor en hijos de madres solteras y en residentes de los estados centrales del país. En otras palabras, el descenso de la tasa general de mortalidad infantil observado en los últimos años ha sido afectado adversamente por ciertas disparidades y favorablemente por otras. El lugar de residencia parece ser el factor que más influye sobre el riesgo de muerte infantil. La multiparidad, la baja escolaridad y el desempleo también son factores importantes en este sentido, específicamente en conexión con la mortalidad posneonatal en el segundo período analizado. Todo esto apunta a la necesidad de reorientar el enfoque de futuras investigaciones, procurando determinar las causas de las diferencias observadas en la mortalidad infantil en los diversos estratos socioeconómicos. (Frank R. Los años de la crisis: an examination of change in differential infant mortality risk within Mexico. Soc Sci Med. 2004;59(4):825-835.)

\section{Disparidades en el tamizaje del cáncer cervicouterino y mamario en mujeres latinas y no latinas en Estados Unidos}

Diversos estudios han revelado disparidades en las tasas de supervivencia y de mortalidad por cáncer de mama y de cuello uterino en mujeres latinas y no latinas en Estados Unidos de América. Las latinas tienen una tasa de mortalidad general por cáncer mamario más baja que las blancas no latinas (15 frente a 27,7 por 100000 , respectivamente), pero una menor tasa de supervivencia a los 5 años $(76 \%$ frente a $85 \%$, respectivamente). Además, entre las latinas el cáncer de mama es la principal causa de mortalidad por cáncer. En cambio, las mujeres latinas tienen una tasa de mortalidad por cáncer cervicouterino más alta que las mujeres blancas que no son latinas (3,4 frente a 2,5 por 100000$)$.

Se cree que las diferencias en las tasas de mortalidad observadas obedecen a factores de riesgo que a su vez se ven acondicionados por diferencias culturales, psicológicas y sociales entre uno y otro grupo de mujeres, por los conocimientos de cada mujer acerca del cáncer y por el momento en que se hace el diagnóstico. Esto último guarda relación con el acceso de las mujeres latinas y de las blancas no latinas a las pruebas de tamizaje: el Papanicolaou en el caso del cáncer de cuello uterino, y la palpación mamaria y la mamografía en el caso del cáncer de mama. Según algunos estudios, las latinas tienen menos probabilidades que las blancas no latinas de haberse sometido a un Papanicolaou, a un examen de palpación mamaria o a una mamografía y, como resultado, el diagnóstico de cáncer en mujeres latinas suele hacerse más tarde que en mujeres blancas que no son latinas.

Se sabe que un menor porcentaje de mujeres latinas tienen seguro médico o asisten con regularidad a un centro de atención sanitaria, en comparación con las mujeres blancas no latinas. La calidad de la atención recibida también podría determinar diferencias en la frecuencia con que se efectúan las pruebas de tamizaje para el cáncer mamario y cervicouterino, pero pocos estudios han examinado este aspecto. En cuanto a posibles conductas desfavorables que podrían ser más frecuentes entre las latinas, los estudios efectuados han arrojado resultados contradictorios. Algunos estudios recientes tampoco han encontrado diferencias en la frecuencia de las pruebas para el tamizaje del cáncer entre latinas y blancas no latinas. Y, si las hubiese, no se sabe en qué medida factores socioeconómicos, la calidad de la atención recibida y el acceso a los servicios de salud podrían estar determinándolas.

Un grupo de investigadores de la Universidad de Columbia, en Nueva York, Estados Unidos de América, se propuso explorar algunos de estos factores y determinar si de hecho hay diferencias entre la población femenina de origen latino y la población de mujeres blancas no latinas en Estados Unidos en lo que respecta a la frecuencia de la mamografía, el examen palpatorio de la mama por el médico y el Papanicolaou en los 12 meses anteriores a la encuesta. Los datos se obtuvieron del suplemento de 1991 correspondiente a Promoción de la Salud y Prevención de Enfermedades de la Encuesta de Salud Nacional por Entrevista (National Health Interview Survey). Con el fin de eliminar la raza como posible factor de confusión, se tomaron en cuenta solamente los datos de mujeres latinas de raza blanca, además de los de las mujeres blancas no latinas. De la encuesta se extrajo información acerca de los hábitos de las mujeres respecto del tamizaje y otros comportamientos relacionados con la salud. Para los análisis en torno a la frecuencia del Papanicolaou se examinaron los datos de 20379 mujeres (1 389 de ellas latinas blancas) mayores de 18 años; para los correspondientes al tamizaje del cáncer de mama, se usaron los datos de 11744 mujeres de 40 años o mayores. Las variables dependientes fueron el haberse sometido a un Papanicolaou, a un examen mamario realizado por un médico y a una mamografía en los últimos 12 meses. Las variables independientes comprendieron la edad, el origen étnico (latino o no latino), el estrato socioeconómico (medido en términos de ingreso familiar y escolaridad), la calidad de la asistencia sanitaria recibida (medida en términos del tipo de proveedor —ninguno, sala de urgencias, hospital para pacien- 
tes ambulatorios, centro de salud y consultorio médico- y de las pruebas efectuadas y preguntas planteadas por el médico durante el último examen físico), y el acceso a atención médica (usando el aseguramiento médico como variable sustituta). Se determinaron las razones de posibilidades (RP) en conexión con el sometimiento a pruebas de tamizaje durante el año anterior a la encuesta; para analizar las diferencias de conducta, así como también el efecto de la calidad de la atención recibida y el aseguramiento, se realizaron regresiones logísticas multifactoriales con ajustes según la edad, escolaridad e ingreso familiar.

Los resultados revelaron que las latinas tenían menos posibilidades de haberse hecho una mamografía en los 12 meses anteriores a la encuesta que las mujeres blancas no latinas ( $R P=0,71$; intervalo de confianza de 95\% [IC95\%]: 0,57-0,88). No obstante, la diferencia se vio atenuada cuando se hicieron ajustes en función de los diversos factores socioeconómicos (RP = 0,90; IC95\%: 0,70-1,15). No se observaron diferencias entre las mujeres blancas latinas y no latinas en cuanto al sometimiento a un Papanicolaou o al examen palpatorio de la mama. Incluso, después de hacer ajustes en función de la edad, factores socioeconómicos y la calidad de la atención, las mujeres latinas se mostraron más propensas que las no latinas a haber recibido un examen palpatorio de la mama. La calidad de la atención así como el acceso a atención médica mostraron valor pronóstico con respecto a la realización de las distintas pruebas de tamizaje. Asimimo, la escolaridad también se vio asociada con las probabilidades de haberse hecho cualquiera de las tres pruebas. Por cada aumento de una unidad en la escolaridad se observó un aumento de $30 \%$ en la frecuencia de mamografías, y de aproximadamente $20 \%$ en la frecuencia de Papanicolaous y exámenes palpatorios de la mama efectuados por profesionales de la salud, una vez que se hicieron ajustes para eliminar el efecto de la edad, el ingreso familiar y el origen étnico.

Tanto la fuente de atención como la minuciosidad del examen físico más reciente tuvieron valor pronóstico en relación con el tamizaje. Esto parece indicar que las mujeres que no reciben una atención óptima no suelen recibir las pruebas de tamizaje de cáncer con la debida frecuencia.

En resumen, estos resultados coinciden con los de otros estudios recientes en que no se ha detectado una asociación, en Estados Unidos, entre el origen étnico latino y una menor frecuencia de pruebas para la detección de cáncer de mama y de cuello uterino. Las asociaciones halladas entre las pruebas y la calidad de la atención y el acceso a ellas tienen implicaciones en lo referente a las políticas de salud. Las propuestas de reforma sanitaria que se han producido en el país no prestan la debida aten- ción a las necesidades de las poblaciones latina y negra, especialmente la de sexo femenino, en la que suele haber más personas pobres. Cabe resaltar que muchas veces se culpa a las personas por tener conductas que se ven determinadas por factores institucionales y sociales. Las desigualdades sociales y la falta de seguro médico son los factores que parecen estar determinando el hecho de que en las mujeres latinas el diagnóstico de cáncer de mama suele hacerse en una etapa más avanzada de la enfermedad que en mujeres blancas no latinas. (Abraído-Lanza AF et al. Breast and cervical cancer screening among latinas and non-latina whites. Am J Public Health 2004;94(8):1393-1398.)

\section{Asociación entre vivir cerca de gasolineras y la leucemia en niños}

La leucemia aguda (LA) es la enfermedad maligna más frecuente en los niños, aunque no es común; su tasa de incidencia es de aproximadamente 4 por 100000 en países desarrollados. Son pocos los factores de riesgo que se han identificado en relación con la leucemia en niños: la trisomía 21, la exposición a dosis elevadas de radiaciones ionizantes y la presencia de ciertas enfermedades raras, tales como la anemia de Fanconi, el síndrome de ataxias-telangiectasias y la neurofibromatosis tipo 1 . Además, diversos estudios epidemiológicos han mostrado una clara asociación entre la leucemia aguda y la exposición ocupacional al benceno en adultos, particularmente en trabajadores de fábricas de caucho, donde el grado de exposición solía ser muy alto anteriormente. En cambio, ningún estudio ha apuntado a una posible asociación entre la leucemia en niños y la exposición al benceno; las concentraciones de benceno en el ambiente son sumamente bajas por comparación con las halladas en el ambiente de las fábricas de caucho. Por este motivo, un grupo de investigadores en Francia llevó a cabo un estudio encaminado a examinar la posibilidad de una asociación entre la exposición ambiental a hidrocarburos y el riesgo de leucemia en la infancia.

El estudio, de casos y testigos, se efectuó en varios hospitales. Se hizo una estratificación de los casos (280 niños con leucemia aguda) y testigos (285 niños sin leucemia) por hospital, edad y sexo. Los datos se obtuvieron mediante entrevistas directas estandarizadas con las madres de los niños que fueron efectuadas por médicos entrenados para esa actividad. Se indagó acerca de cualquier posible exposición a hidrocarburos en las distintas viviendas que hubiese ocupado la familia del niño desde el momento en que este fue concebido hasta la fecha del diagnóstico de leucemia o de la entrevista (en el caso de los testigos). La exposición in utero y la ex- 